\title{
Dissipativity of the backward Euler method for nonlinear Volterra functional differential equations in Banach space
}

Siqing Gan

\section{"Correspondence:}

sqgan@csu.edu.cn

School of Mathematics and

Statistics, Central South University,

Changsha, Hunan 410083, China

\section{Springer}

\begin{abstract}
This paper concerns the dissipativity of nonlinear Volterra functional differential equations (VFDEs) in Banach space and their numerical discretization. We derive sufficient conditions for the dissipativity of nonlinear VFDEs. The general results provide a unified theoretical treatment for dissipativity analysis to ordinary differential equations (ODEs), delay differential equations (DDEs), integro-differential equations (IDEs) and VFDEs of other type appearing in practice. Then the dissipativity property of the backward Euler method for VFDEs is investigated. It is shown that the method can inherit the dissipativity of the underlying system. The close relationship between the absorbing set of the numerically discrete system generated by the backward Euler method and that of the underlying system is revealed.
\end{abstract}

Keywords: dissipativity; Volterra functional differential equation; Banach space; backward Euler method

\section{Introduction}

Many dynamical systems are characterized by the property of possessing a bounded absorbing set where all trajectories enter in a finite time and thereafter remain inside. Such systems are called dissipative. Dissipativity means that the eventual time evolution of solutions is confined to a bounded absorbing set. In the study of numerical methods, it is natural to ask whether those discrete systems preserve the dissipativity of the continuous system.

Since the 1990s considerable process has been made in dissipativity analysis of numerical methods. The papers [1-5] focus on the numerical methods for ordinary differential equations. For the delay differential equations (DDEs) with constant delay, sufficient conditions for the dissipativity of analytical and numerical solutions are presented in [6-8]. Since that, the analysis is extended to DDEs with variable lags $[9,10]$ and Volterra functional differential equations [11-16].

The dissipativity analysis of numerical methods for VFDEs in the literature was limited in Euclidean spaces or Hilbert spaces. The aim of this paper is to investigate the dissipativity of nonlinear VFDEs in Banach space and their numerical discretization.

The main contributions of this paper could be summarized as follows.

(c) 2015 Gan; licensee Springer. This article is distributed under the terms of the Creative Commons Attribution 4.0 International License (http://creativecommons.org/licenses/by/4.0/), which permits unrestricted use, distribution, and reproduction in any medium, provided you give appropriate credit to the original author(s) and the source, provide a link to the Creative Commons license, and indicate if changes were made. 
(a) Sufficient conditions for the dissipativity of nonlinear VFDEs in Banach space are derived. The general results provide a unified theoretical treatment for dissipativity analysis to ordinary differential equations (ODEs), delay differential equations (DDEs), integrodifferential equations (IDEs) and VFDEs of other type appearing in practice. In particular, the theory covers the existing dissipativity results of DDEs with a wide variety of delay arguments such as constant delays, bounded and unbounded vary delays, discrete and distributed delays and so on.

(b) It is proved that the backward Euler method can inherit the dissipativity of the underlying system. Theorem 4.1 and Theorem 4.2 show the close relationship between the absorbing set of VFDEs and that of the numerically discrete system generated by the backward Euler method. It implies that the radius of the absorbing set of the discrete system approaches to that of the underlying system as the stepsize approaches to zero. On the contrary, most of the existing dissipativity results of numerical methods for VFDEs are independent of the size of the absorbing set of the underlying system.

This paper is organized as follows. In Section 2, some basic concepts for nonlinear VFDEs in Banach space are presented. In Section 3, some sufficient conditions for the dissipativity of nonlinear VFDEs are given. In Section 4, it is shown that the backward Euler method can inherit the dissipativity of the underlying system.

\section{Some concepts}

Let $X$ be a real or complex Banach space with the norm $\|\cdot\|$. For any given closed interval $I \subset \mathbb{R}$, let the symbol $C_{X}(I)$ denote a Banach space consisting of all continuous mappings $x: I \rightarrow X$, on which the norm is defined by $\|x\|_{\infty}=\max _{t \in I}\|x(t)\|$.

Consider the following initial value problem (IVP) [17]:

$$
\begin{cases}y^{\prime}(t)=f(t, y(t), y), & t \geq a, \\ y(t)=\varphi(t), & a-\tau \leq t \leq a,\end{cases}
$$

where $a, \tau$ are constants, $0 \leq \tau \leq+\infty, \varphi \in C_{X}[a-\tau, a]$ is a given initial function, $f$ : $[a,+\infty) \times X \times C_{X}[a-\tau,+\infty) \rightarrow X$ is a given continuous mapping satisfying the conditions

$$
\begin{aligned}
& (1-\lambda \alpha(t)) D_{f}(0, t, u, \psi) \\
& \leq D_{f}(\lambda, t, u, \psi)+\lambda\left(\gamma(t)+\beta(t) \max _{t-\mu_{2}(t) \leq \xi \leq t-\mu_{1}(t)}\|\psi(\xi)\|^{2}\right), \\
& \forall \lambda \geq 0, t \in[a,+\infty), u \in X, \psi \in C_{X}[a-\tau,+\infty),
\end{aligned}
$$

where

$$
D_{f}(\lambda, t, u, \psi)=\|u-\lambda f(t, u, \psi)\|^{2}
$$

Here $\alpha(t), \beta(t), \gamma(t)$ are continuous functions, $\mu_{1}(t)$ and $\mu_{2}(t)$ satisfy

$$
0 \leq \mu_{1}(t) \leq \mu_{2}(t) \leq t-a+\tau, \quad \forall t \in[a,+\infty) .
$$


Define

$$
\begin{aligned}
& \mu_{1}^{(0)}=\inf _{a \leq t<\infty} \mu_{1}(t) \geq 0, \quad \mu_{2}^{(0)}\left(\xi_{1}, \xi_{2}\right)=\inf _{\xi_{1} \leq t \leq \xi_{2}}\left(t-\mu_{2}(t)\right) \geq a-\tau, \\
& \forall \xi_{1}, \xi_{2}: a \leq \xi_{1} \leq \xi_{2}<+\infty .
\end{aligned}
$$

We always assume that problem (2.1) has a unique solution on the interval $[a-\tau,+\infty)$. Condition (2.2) implies that the mapping $f(t, \psi(t), \psi)$ is independent of the values of the function $\psi(\xi)$ with $t<\xi \leq b$, i.e., $f(t, \psi(t), \psi)$ is a Volterra functional.

For simplicity, we use the symbol $\mathcal{A}\left(\alpha, \beta, \gamma, \mu_{1}, \mu_{2}\right)$ to denote the problem class consisting of all problems (2.1) satisfying condition (2.2).

For the special case where $X$ is a Hilbert space with the inner product $\langle\cdot, \cdot\rangle$ and the corresponding norm $\|\cdot\|$, condition (2.2) is equivalent to

$$
\begin{aligned}
& 2 \operatorname{Re}\langle u, f(t, u, \psi)\rangle \leq \gamma(t)+\alpha(t)\|u\|^{2}+\beta(t) \max _{t-\mu_{2}(t) \leq \xi \leq t-\mu_{1}(t)}\|\psi(\xi)\|^{2}, \\
& \forall t \in[a,+\infty), u \in X, \psi \in C_{X}[a-\tau,+\infty) .
\end{aligned}
$$

The dissipativity analysis of $(2.6)$ can be found in $[14,16]$.

\section{Dissipativity of nonlinear Volterra functional differential equations}

Definition 3.1 [6] The evolutionary equation (2.1) is said to be dissipative in $X$ if there is a bounded set $\mathcal{B} \subset X$ such that for all bounded sets $\Psi \subset X$ there is a time $t_{0}=t_{0}(\Psi)$ such that for all initial functions $\varphi(t)$ contained in $\Psi$, the corresponding solution $y(t)$ is contained in $\mathcal{B}$ for all $t \geq t_{0}$. $\mathcal{B}$ is called an absorbing set in $X$.

Lemma 3.2 Equation (2.2) implies that $\gamma(t) \geq 0$ and $\beta(t) \geq 0$.

Proof Setting $u \equiv 0$ in (2.2) we obtain

$$
\begin{gathered}
0 \leq \lambda\|f(t, 0, \psi)\|^{2}+\gamma(t)+\beta(t) \max _{t-\mu_{2}(t) \leq \xi \leq t-\mu_{1}(t)}\|\psi(\xi)\|^{2}, \\
\forall \lambda>0, t \in[a,+\infty), \psi \in C_{X}[a-\tau,+\infty),
\end{gathered}
$$

which yields

$$
0 \leq \gamma(t)+\beta(t) \max _{t-\mu_{2}(t) \leq \xi \leq t-\mu_{1}(t)}\|\psi(\xi)\|^{2}, \quad \forall t \in[a,+\infty), \psi \in C_{X}[a-\tau,+\infty)
$$

as $\lambda \rightarrow 0$. Let $\psi(t) \equiv 0$. It follows from (3.1) that $\gamma(t) \geq 0$. If there is $\tilde{t} \geq a$ such that $\beta(\tilde{t})<0$, it is easy to find a function $\psi \in C_{X}[a-\tau,+\infty)$ which satisfies

$$
\gamma(\tilde{t})+\beta(\tilde{t}) \max _{\tilde{t}-\mu_{2}(\tilde{t}) \leq \xi \leq \tilde{t}-\mu_{1}(\tilde{t})}\|\psi(\xi)\|^{2}<0,
$$

which contradicts (3.1). Therefore, $\beta(t) \geq 0$.

For a continuous real-valued function $y(t)$ of a real variable, the Dini derivatives $D^{+} y(t)$ and $D_{-} y(t)$ are defined as

$$
D^{+} y(t)=\limsup _{\delta \searrow 0} \frac{y(t+\delta)-y(t)}{\delta} \quad \text { and } \quad D_{-} y(t)=\liminf _{\delta \succ 0} \frac{y(t+\delta)-y(t)}{\delta} .
$$


Lemma 3.3 If $u(t) \geq 0, t \in(-\infty,+\infty)$, and

$$
\begin{aligned}
& u^{\prime}(t) \leq \gamma(t)+\alpha(t) u(t)+\beta(t) \sup _{t-\tau(t) \leq \xi \leq t} u(\xi), \quad t \geq a, \\
& u(t)=|\psi(t)|, \quad t \leq a,
\end{aligned}
$$

where $\psi(t)$ is bounded and continuous for $t \leq a$, continuous functions $\gamma(t) \geq 0, \beta(t) \geq 0$ and $\alpha(t)<0$ for $t \in[a,+\infty), \tau(t) \geq 0$ and

$$
\lim _{t \rightarrow+\infty}(t-\tau(t)) \rightarrow+\infty, \quad \sup _{a \leq t<+\infty} \alpha(t)<0, \quad \sup _{a \leq t<+\infty} \frac{\beta(t)}{|\alpha(t)|}<1 .
$$

Then, for any given $\epsilon>0$, there exists $\hat{t}=\hat{t}(G, \epsilon)>a$ such that

$$
u(t) \leq \frac{\gamma^{*}}{\sigma}+\epsilon, \quad t \geq \hat{t}
$$

where

$$
\gamma^{*}=\sup _{a \leq t<+\infty} \gamma(t), \quad G=\sup _{-\infty<\xi \leq a}|\psi(\xi)|
$$

Proof The last two inequalities of (3.3) imply that (2.10) and (2.12) of [16] hold. The conclusion follows from Theorem 2.3 of [16] directly.

Theorem 3.4 Suppose problem $(2.1) \in \mathcal{A}\left(\alpha, \beta, \gamma, \mu_{1}, \mu_{2}\right)$ and that

$$
\lim _{t \rightarrow+\infty}\left(t-\mu_{2}(t)\right) \rightarrow+\infty, \quad \sup _{a \leq t<+\infty} \alpha(t)<0, \quad \sup _{a \leq t<+\infty} \frac{\beta(t)}{|\alpha(t)|}<1 .
$$

Then, for any given $\epsilon>0$, there exists $\check{t}=\check{t}(\bar{\varphi}, \epsilon)$ such that

$$
\|y(t)\|^{2} \leq \frac{\gamma^{*}}{\sigma}+\epsilon, \quad t>\check{t}
$$

where $\gamma^{*}=\sup _{t \geq a} \gamma(t), \bar{\varphi}=\sup _{t \leq a}\|\varphi(t)\|^{2}$. Hence the system is dissipative with an absorbing set $B=B\left(0, \sqrt{\gamma^{*} / \sigma+\epsilon}\right)$.

Proof By the definition of Dini derivative, we have

$$
\begin{aligned}
D_{-}\left(\|y(t)\|^{2}\right) & =\liminf _{\delta \rightarrow-0} \frac{\|y(t+\delta)\|^{2}-\|y(t)\|^{2}}{\delta} \\
& =\liminf _{\delta \rightarrow-0} \frac{\|y(t+\delta)\|-\|y(t)\|}{\delta}(\|y(t+\delta)\|+\|y(t)\|), \\
D^{+}\left(\|y(t)\|^{2}\right) & =\liminf _{\delta \rightarrow+0} \frac{\|y(t+\delta)\|^{2}-\|y(t)\|^{2}}{\delta} \\
& =\liminf _{\delta \rightarrow+0} \frac{\|y(t+\delta)\|-\|y(t)\|}{\delta}(\|y(t+\delta)\|+\|y(t)\|) .
\end{aligned}
$$


Applying Lemma 4.6.2 of [18], we see that the limits

$$
\lim _{\delta \rightarrow-0} \frac{\left\|y(t)+\delta y^{\prime}(t)\right\|-\|y(t)\|}{\delta} \text { and } \lim _{\delta \rightarrow+0} \frac{\left\|y(t)+\delta y^{\prime}(t)\right\|-\|y(t)\|}{\delta}
$$

exist. Lemma 4.6.3 of [18] tells us that

$$
\begin{aligned}
& \liminf _{\delta \rightarrow-0} \frac{\|y(t+\delta)\|-\|y(t)\|}{\delta}=\lim _{\delta \rightarrow-0} \frac{\left\|y(t)+\delta y^{\prime}(t)\right\|-\|y(t)\|}{\delta}, \\
& \liminf _{\delta \rightarrow+0} \frac{\|y(t+\delta)\|-\|y(t)\|}{\delta}=\lim _{\delta \rightarrow+0} \frac{\left\|y(t)+\delta y^{\prime}(t)\right\|-\|y(t)\|}{\delta} .
\end{aligned}
$$

Then (3.7) and (3.8) together imply that $D_{-}\left(\|y(t)\|^{2}\right)=D^{+}\left(\|y(t)\|^{2}\right)$. Let $u(t)=\|y(t)\|^{2}$. Therefore, $u^{\prime}(t)$ exists, and

$$
\begin{aligned}
u^{\prime}(t) & =\lim _{\delta \rightarrow-0} \frac{\|y(t+\delta)\|^{2}-\|y(t)\|^{2}}{\delta} \\
& =\lim _{\delta \rightarrow-0} \frac{\left\|y(t)+\delta y^{\prime}(t)+o(\delta)\right\|^{2}-\left\|y(t)+\delta y^{\prime}(t)\right\|^{2}+\left\|y(t)+\delta y^{\prime}(t)\right\|^{2}-\|y(t)\|^{2}}{\delta} \\
& =\lim _{\delta \rightarrow-0} \frac{\left\|y(t)+\delta y^{\prime}(t)\right\|^{2}-\|y(t)\|^{2}}{\delta} \\
& =\lim _{\delta \rightarrow-0} \frac{\|y(t)+\delta f(t, y(t), y)\|^{2}-\|y(t)\|^{2}}{\delta} .
\end{aligned}
$$

Using condition (2.2), we have

$$
\begin{aligned}
u^{\prime}(t) & \leq \lim _{\delta \rightarrow-0} \frac{(1+\delta \alpha(t))\|y(t)\|^{2}+\delta\left(\gamma(t)+\beta(t) \max _{t-\mu_{2}(t) \leq \xi \leq t-\mu_{1}(t)}\|y(\xi)\|^{2}\right)-\|y(t)\|^{2}}{\delta} \\
& =\gamma(t)+\alpha(t)\|y(t)\|^{2}+\beta(t) \max _{t-\mu_{2}(t) \leq \xi \leq t-\mu_{1}(t)}\|y(\xi)\|^{2},
\end{aligned}
$$

that is,

$$
u^{\prime}(t) \leq \gamma(t)+\alpha(t) u(t)+\beta(t) \max _{t-\mu_{2}(t) \leq \xi \leq t-\mu_{1}(t)} u(\xi) .
$$

The desired result follows from Theorem 3.3. The proof is complete.

Remark 3.5 Specializing Theorem 3.4 to Hilbert spaces, we can obtain the corresponding result which is in accordance with that obtained in [16].

Remark 3.6 Specializing Theorem 3.4 to DDEs in Hilbert spaces with constant delays and $\alpha(t) \equiv \alpha, \beta(t) \equiv \beta, \gamma(t) \equiv \gamma$, we can obtain the corresponding result which is in accordance with that obtained in [6].

Remark 3.7 Specializing Theorem 3.4 to ODEs in Euclidean spaces with $\alpha(t) \equiv \alpha$, $\gamma(t) \equiv \gamma$, we can obtain the corresponding result which is in accordance with that obtained in [4].

Remark 3.8 Theorem 3.4 covers most of the existing dissipativity results of DDEs with a wide variety of delay arguments such as constant delays [6], bounded varying delays 
[10] and unbounded varying delays [9], discrete and distributed delays [11, 12] and so on. In brief, Theorem 3.4 provides a unified theoretical treatment for dissipativity analysis to ordinary differential equations (ODEs), delay differential equations (DDEs), integrodifferential equations (IDEs) and VFDEs of other type appearing in practice.

\section{Dissipativity of the backward Euler method}

For simplicity, from now on we assume that

$$
\gamma(t) \equiv \gamma, \quad \alpha(t) \equiv \alpha, \quad \beta(t) \equiv \beta, \quad t \in[a,+\infty) .
$$

Theorem 3.4 can be rewritten as follows.

Theorem 4.1 Suppose problem $(2.1) \in \mathcal{A}\left(\alpha, \beta, \gamma, \mu_{1}, \mu_{2}\right)$ and that

$$
\lim _{t \rightarrow+\infty}\left(t-\mu_{2}(t)\right) \rightarrow+\infty, \quad \alpha+\beta<0
$$

Then, for any given $\epsilon>0$, there exist $\check{t}=\check{t}(\bar{\varphi}, \epsilon), \bar{\varphi}=\sup _{t \leq a}\|\varphi(t)\|^{2}$ such that

$$
\|y(t)\| \leq \sqrt{\frac{\gamma}{-(\alpha+\beta)}+\epsilon}, \quad t>\check{t} .
$$

The backward Euler method applied to (2.1) gives

$$
\begin{cases}y_{n+1}=y_{n}+h f\left(t_{n+1}, y_{n+1}, y^{h}(\cdot)\right), & n=0,1,2, \ldots \\ y^{h}(t)=\pi^{h}\left(t, \varphi, y_{1}, y_{2}, \ldots, y_{n+1}\right), & a-\tau \leq t \leq t_{n+1}\end{cases}
$$

where $\pi^{h}$ is an appropriate interpolation operator which approximates to the exact solution $y(t)$ on the interval $[a-\tau, b], h>0$ is the stepsize, $y_{n}$ is an approximation to the exact solution $y\left(t_{n}\right)$ with $t_{n}=a+n h$.

Noting that the backward Euler method for ODEs is of order one, we can use the following piecewise linear interpolation:

$$
y^{h}(t)= \begin{cases}\frac{1}{h}\left[\left(t_{i+1}-t\right) y_{i}+\left(t-t_{i}\right) y_{i+1}\right], & t_{i} \leq t \leq t_{i+1} \\ \varphi(t), & a-\tau \leq t \leq a\end{cases}
$$

Theorem 4.2 Assume that problem $(2.1) \in \mathcal{A}\left(\alpha, \beta, \gamma, \mu_{1}, \mu_{2}\right)$ and that

$$
\lim _{t \rightarrow+\infty}\left(t-\mu_{2}(t)\right) \rightarrow+\infty, \quad \alpha+\beta<0
$$

Let $\left\{y_{n}\right\}$ be the sequence of numerical solutions obtained by (4.1)-(4.2). Then, for any given $\epsilon>0$, there exists $n_{0}=n_{0}(\bar{\varphi}, \epsilon)$ such that

$$
\left\|y_{n}\right\| \leq \sqrt{\frac{\gamma}{-(\alpha+\beta)} \cdot \frac{1-h \alpha}{1-h(\alpha+\beta)}+\epsilon}, \quad n \geq n_{0} .
$$

Proof It follows from (4.1) that

$$
\left\|y_{n+1}-h f\left(t_{n+1}, y_{n+1}, y^{h}(\cdot)\right)\right\|^{2}=\left\|y_{n}\right\|^{2}
$$


Using (2.2), we have

$$
\begin{aligned}
& \left\|y_{n+1}-h f\left(t_{n+1}, y_{n+1}, y^{h}(\cdot)\right)\right\|^{2} \\
& \quad \geq(1-h \alpha)\left\|y_{n+1}\right\|^{2}-h\left(\gamma+\beta \max _{t_{n+1}-\mu_{2}\left(t_{n+1}\right) \leq \xi \leq t_{n+1}-\mu_{1}\left(t_{n+1}\right)}\left\|y^{h}(\xi)\right\|^{2}\right) .
\end{aligned}
$$

It follows from (4.3) and (4.4) that

$$
(1-h \alpha)\left\|y_{n+1}\right\|^{2} \leq\left\|y_{n}\right\|^{2}+h\left(\gamma+\beta \max _{t_{n+1}-\mu_{2}\left(t_{n+1}\right) \leq \xi \leq t_{n+1}-\mu_{1}\left(t_{n+1}\right)}\left\|y^{h}(\xi)\right\|^{2}\right) .
$$

In view of (4.2), we have

$$
\max _{t_{n+1}-\mu_{2}\left(t_{n+1}\right) \leq \xi \leq t_{n+1}-\mu_{1}\left(t_{n+1}\right)}\left\|y^{h}(\xi)\right\|^{2} \leq \max \left\{\max _{1 \leq i \leq n+1}\left\|y_{i}\right\|^{2}, \max _{a-\tau \leq t \leq a}\|\varphi(t)\|^{2}\right\},
$$

where we used the following inequality:

$$
\left\|(1-\delta) y_{i}+\delta y_{i+1}\right\|^{2} \leq(1-\delta)\left\|y_{i}\right\|^{2}+\delta\left\|y_{i+1}\right\|^{2} \leq \max \left\{\left\|y_{i}\right\|^{2},\left\|y_{i+1}\right\|^{2}\right\}
$$

A combination of (4.5) and (4.6) leads to

$$
(1-h \alpha)\left\|y_{n+1}\right\|^{2} \leq\left\|y_{n}\right\|^{2}+h\left(\gamma+\beta \max \left\{\max _{1 \leq i \leq n+1}\left\|y_{i}\right\|^{2}, \max _{a-\tau \leq t \leq a}\|\varphi(t)\|^{2}\right\}\right) .
$$

For simplicity, for any given nonnegative integer $n$, we write

$$
\begin{aligned}
Q_{n} & =\max \left\{\max _{1 \leq i \leq n}\left\|y_{i}\right\|^{2}, \max _{a-\tau \leq t \leq a}\|\varphi(t)\|^{2}\right\}, \quad n \geq 1, \\
Q_{0} & =\max _{a-\tau \leq t \leq a}\|\varphi(t)\|^{2} .
\end{aligned}
$$

We now consider two cases:
(a) $\max \left\{\max _{1 \leq i \leq n+1}\left\|y_{i}\right\|^{2}, \max _{a-\tau \leq t \leq a}\|\varphi(t)\|^{2}\right\}=\left\|y_{n+1}\right\|^{2}$,
(b) $\max \left\{\max _{1 \leq i \leq n+1}\left\|y_{i}\right\|^{2}, \max _{a-\tau \leq t \leq a}\|\varphi(t)\|^{2}\right\} \neq\left\|y_{n+1}\right\|^{2}$.

In the case of (a), it follows from (4.7) that

$$
\left\|y_{n+1}\right\|^{2} \leq \frac{h \gamma}{1-h(\alpha+\beta)}+\frac{1}{1-h(\alpha+\beta)}\left\|y_{n}\right\|^{2} .
$$

In the case of (b), it follows from (4.7) that

$$
\left\|y_{n+1}\right\|^{2} \leq \frac{h \gamma}{1-h \alpha}+\frac{1}{1-h \alpha}\left(\left\|y_{n}\right\|^{2}+h \beta Q_{n}\right) .
$$

To summarize both of the two cases, we have shown that

$$
\left\|y_{n+1}\right\|^{2} \leq \max \left\{\frac{h \gamma}{1-h(\alpha+\beta)}, \frac{h \gamma}{1-h \alpha}\right\}+\max \left\{\frac{1}{1-h(\alpha+\beta)}, \frac{1+h \beta}{1-h \alpha}\right\} Q_{n},
$$


which yields

$$
\left\|y_{n+1}\right\|^{2} \leq \frac{h \gamma}{1-h(\alpha+\beta)}+\frac{1+h \beta}{1-h \alpha} Q_{n}=: d_{0}+d_{1} Q_{n}
$$

where

$$
d_{0}=\frac{h \gamma}{1-h(\alpha+\beta)}, \quad d_{1}=\frac{1+h \beta}{1-h \alpha}<1 .
$$

Considering $Q_{n}=\left\|y_{n}\right\|^{2}$ or $Q_{n} \neq\left\|y_{n}\right\|^{2}$ and inserting (4.10) repeatedly, we obtain

$$
\begin{aligned}
\left\|y_{n}\right\|^{2} & \leq d_{0}\left(1+d_{1}+\cdots+d_{1}^{n-1}\right)+d_{1}^{n} Q_{0} \\
& \leq \frac{d_{0}}{1-d_{1}}+d_{1}^{n} Q_{0}=\frac{\gamma}{-(\alpha+\beta)} \frac{1-h \alpha}{1-h(\alpha+\beta)}+d_{1}^{n} Q_{0} .
\end{aligned}
$$

Therefore, for any given $\epsilon>0$, there exists $n_{0}=n_{0}(\bar{\varphi}, \epsilon)$ such that

$$
\left\|y_{n}\right\| \leq \sqrt{\frac{\gamma}{-(\alpha+\beta)} \cdot \frac{1-h \alpha}{1-h(\alpha+\beta)}+\epsilon}, \quad n \geq n_{0} .
$$

This completes the proof of the theorem.

Remark 4.3 Theorem 4.1 and Theorem 4.2 show the close relationship between the absorbing set of the underlying system and that of the numerically discrete system generated by the backward Euler method. On the contrary, most of the existing dissipativity results of numerical methods for VFDEs are independent of the size of the absorbing set of the underlying system. It is obvious that the radius of the absorbing set of the discrete system is longer than that of the underlying system because of $\frac{1-h \alpha}{1-h(\alpha+\beta)} \geq 1$. Furthermore, Theorem 4.2 implies the following facts.

- In the case of ODEs, that is $\beta=0$, for any given $\epsilon>0$, the ball $B\left(0, \sqrt{\frac{\gamma}{-\alpha}+\epsilon}\right)$ is an absorbing set of the discrete system as well as the underlying system. The absorbing set is independent of the stepsize of the backward Euler method.

- For fixed $\beta>0, h>0$, if $|\alpha|$ is sufficiently large, then the difference between the radius of the absorbing set of the numerically discrete system and that of the underlying system is sufficiently small.

- Notice that $\frac{1-h \alpha}{1-h(\alpha+\beta)} \rightarrow 1$ as $h \rightarrow 0$, hence given any $\epsilon>0$, there exists $h_{0}=h_{0}(\epsilon)$ such that for $h<h_{0}$ the ball $B\left(0, \sqrt{\frac{\gamma}{-(\alpha+\beta)}+\epsilon}\right)$ is an absorbing set of the discrete system. In other words, the radius of the absorbing set of the discrete system approaches to that of the underlying system as the stepsize approaches to zero. 


\section{References}

1. Hill, AT: Global dissipativity for A-stable methods. SIAM J. Numer. Anal. 34, 119-142 (1997)

2. Hill, AT: Dissipativity of Runge-Kutta methods in Hilbert spaces. BIT Numer. Math. 37, 37-42 (1997)

3. Humphries, AR, Stuart, AM: Model problems in numerical stability theory for initial value problems. SIAM Rev. 36, 226-257 (1994)

4. Humphries, AR, Stuart, AM: Runge-Kutta methods for dissipative and gradient dynamical systems. SIAM J. Numer. Anal. 31, 1452-1485 (1994)

5. Xiao, A: Dissipativity of general linear methods for dissipative dynamical systems in Hilbert spaces. Math. Numer. Sin. 22, 429-436 (2000) (in Chinese)

6. Huang, C: Dissipativity of Runge-Kutta methods for dissipative systems with delays. IMA J. Numer. Anal. 20, 153-166 (2000)

7. Huang, C: Dissipativity of one-leg methods for dissipative systems with delays. Appl. Numer. Math. 35, 11-22 (2000)

8. Huang, C, Chang, Q: Dissipativity of multistep Runge-Kutta methods for dynamical systems with delays. Math. Comput. Model. 40(11-12), 1285-1296 (2004)

9. Gan, S: Exact and discretized dissipativity of the pantograph equation. J. Comput. Math. 25(1), 81-88 (2007)

10. Tian, $\mathrm{H}$ : Numerical and analytic dissipativity of the $\theta$-method for delay differential equations with a bounded lag. Int. J. Bifurc. Chaos 14, 1839-1845 (2004)

11. Gan, S: Dissipativity of linear $\theta$-methods for integro-differential equations. Comput. Math. Appl. 52, $449-458$ (2006)

12. Gan, S: Dissipativity of $\theta$-methods for nonlinear Volterra delay-integro-differential equations. J. Comput. Appl. Math. 206, 898-907 (2007)

13. Tian, H, Guo, N: Asymptotic stability, contractivity and dissipativity of one-leg $\theta$-method for non-autonomous delay functional differential equations. Appl. Math. Comput. 203, 333-342 (2008)

14. Wen, L: Numerical stability analysis for nonlinear Volterra functional differential equations in abstract spaces. Ph.D. thesis, Xiangtan University (2005) (in Chinese)

15. Wen, L, Li, S: Dissipativity of Volterra functional differential equations. J. Math. Anal. Appl. 324(1), $696-706$ (2006)

16. Wen, L, Yu, Y, Wang, W: Generalized Halanay inequalities for dissipativity of Volterra functional differential equations. J. Math. Anal. Appl. 347, 169-178 (2008)

17. Li, S: Stability analysis of solutions to nonlinear stiff Volterra functional differential equations in Banach space. Sci. China Ser. A 48, 372-387 (2005)

18. Li, S: Theory of Computational Methods for Stiff Differential Equations. Hunan Science and Technology Publisher, Changsha (1997) (in Chinese) 